\title{
Spin-Ordering Quantum Transitions of Superconductors in a Magnetic Field
}

\section{Citation}

Demler, Eugene, Subir Sachdev, and Ying Zhang. 2001. "Spin-Ordering Quantum Transitions of Superconductors in a Magnetic Field." Physical Review Letters 87 (6) (July 19). doi:10.1103/ physrevlett.87.067202.

\section{Published Version}

doi:10.1103/PhysRevLett.87.067202

\section{Permanent link}

http://nrs.harvard.edu/urn-3:HUL.InstRepos:27989891

\section{Terms of Use}

This article was downloaded from Harvard University's DASH repository, and is made available under the terms and conditions applicable to Other Posted Material, as set forth at http:// nrs.harvard.edu/urn-3:HUL.InstRepos:dash.current.terms-of-use\#LAA

\section{Share Your Story}

The Harvard community has made this article openly available.

Please share how this access benefits you. Submit a story.

\section{Accessibility}




\title{
Spin-Ordering Quantum Transitions of Superconductors in a Magnetic Field
}

\author{
Eugene Demler, ${ }^{1}$ Subir Sachdev, ${ }^{1,2}$ and Ying Zhang ${ }^{2}$ \\ ${ }^{1}$ Department of Physics, Harvard University, Cambridge, Massachusetts 02138 \\ ${ }^{2}$ Department of Physics, Yale University, P.O. Box 208120, New Haven, Connecticut 06520-8120
}

(Received 20 March 2001; published 19 July 2001)

\begin{abstract}
We argue that recent neutron scattering measurements by Lake et al. [Science 291, 1759 (2001)] of the spin excitation spectrum of $\mathrm{La}_{2}-\delta \mathrm{Sr}_{\delta} \mathrm{CuO}_{4}$ in a magnetic field can be understood in terms of proximity to a phase with co-existing superconductivity and spin density wave order. We present a general theory for such quantum transitions, and argue that their low energy spin fluctuations are controlled by a singular correction from the superflow kinetic energy, acting in the region outside the vortex cores. We propose numerous experimental tests of our theory.
\end{abstract}

PACS numbers: 75.10.Jm, 74.25.-q, 74.60.-w, 76.50.+g

Recent neutron scattering experiments of Lake et al. [1] have opened a new window on the spin excitation spectrum of the high temperature superconductors. They have observed that a moderate magnetic field induces an anomalously large increase in the spectral density of low energy spin fluctuations in $\mathrm{La}_{2-\delta} \mathrm{Sr}_{\delta} \mathrm{CuO}_{4}$ at optimal doping $(\delta=0.163)$ and low temperatures $(T)$. Experiments on the underdoped $\mathrm{La}_{2-\delta} \mathrm{Sr}_{\delta} \mathrm{CuO}_{4}$ also show a large increase in the intensity of elastic neutron scattering in the applied magnetic field [2-4]. There have been a number of studies of enhanced antiferromagnetic correlations in the cores of vortices in the superconducting order [5-7], and one interpretation of the measurements is that the extra scattering arises from quasistatic moments [6,7] in the cores of the field-induced vortices. The measurements then lead to the estimate [1] that each $\mathrm{Cu}$ site in the vortex core has a moment of order $0.6 \mu_{B}$ in the anomalous low energy sector. Such a large moment is characteristic of the insulating antiferromagnet $(\delta=0)$, and would require a corresponding charge disproportionation with a large Coulomb energy cost; this is a difficulty with such an interpretation.

We argue here that the experiments can be understood by assuming that the superconductor (SC) is in the vicinity of a bulk quantum phase transition to a state with microscopic coexistence of SC and spin density wave (SDW) orders; the latter state has been considered in a number of studies [8-14], and has been observed in excess-oxygendoped $\mathrm{La}_{2} \mathrm{CuO}_{4+y}$ [15]. The magnetic field, $H$, drives the SC phase closer to the SC + SDW phase-see Fig. 1. Initially, $H$ induces well separated vortices in the SC, and there could be small additional magnetic scattering from relatively high energy spin $S=1$ excitons centered around the vortex cores. However, with increasing $H$ [but with $H / H_{c 2}^{0}$ still small $\left(H_{c 2}^{0}\right.$ is the upper critical field at which the mixed vortex state disappears for a particular set of parameters - see Fig. 1)] the energy of any such exciton decreases rapidly, and it becomes part of a delocalized collective spin fluctuation which is a precursor of the transition to the SC + SDW phase; the dominant magnetic scattering then arises from the region outside the vortex cores. Our primary results for extreme type-II superconductors [Ginzburg-Landau (GL) parameter $\kappa \rightarrow \infty$ ] are as follows: (i) for small $H / H_{c 2}^{0}$, the $\mathrm{SC}$ to $\mathrm{SC}+\mathrm{SDW}$ phase boundary (MA in Fig. 1; the caption defines $r, r_{c}$ ) is at $H \sim\left(r-r_{c}\right) / \ln \left[1 /\left(r-r_{c}\right)\right]$ - so this boundary is anomalously flat at small $H$, and this allows the system to move close to it for not too large $H$; (ii) the energy,



FIG. 1. Phase diagram of $S+F_{\mathrm{GL}} / T$ at $T=0$ and large $N$ as a function of the field $H$ and the parameter $r$ (which is similar, but not identical to the doping $\delta$ ) in the limit $\kappa \rightarrow \infty$. This theory offers a complete, experimentally applicable, description only of the SC to SC + SDW transition at small $H$, away from the tetracritical point $\mathrm{M}$. The remaining phase diagram is qualitative, and the non-SC phases should have some additional charge ordering. The path of the experiments of [1] is denoted by the vertical arrow. The upper critical field of the SC state clearly depends upon $r$, and $H_{c 2}^{0}$ is its value at $M$. The point $\mathrm{A}$ is at the nonuniversal value $r=r_{c}$, but the remaining phase boundaries can be expressed in terms of $r_{c}$ and the couplings in $S$ [21]. The point $\mathrm{M}$ is at $H / H_{c 2}^{0}=1, r=r_{c}+v$, the boundary BM is at $H / H_{c 2}^{0}=1-\left[v^{2}+v\left(r_{c}-r\right)\right] / 4 u, \mathrm{CM}$ is at $r=r_{c}+v$, and DM is at $H / H_{c 2}^{0}=1+N v \Delta /\left(8 \pi c^{2}\right)$, where $\Delta$ is the solution of $\Delta^{2}+N u /\left(2 \pi c^{2}\right) \Delta+v-r+r_{c}=0$. Near M, the position of $\mathrm{AM}$ is given by $H / H_{c 2}^{0}=1-\vartheta_{1}\left(r_{c}+v-r\right) / v$ where $\vartheta_{1}=1.1596-\mathcal{O}\left(v^{2}\right)\left[\vartheta_{1}=1.1803-\mathcal{O}\left(v^{2}\right)\right]$ for a triangular (square) Abrikosov flux lattice [the $\mathcal{O}\left(v^{2}\right)$ terms will be described elsewhere]. The small $H$ behavior of AM is one of our main results, and is in (10). 
$\epsilon_{00}(H)$, of the low energy peak in the neutron scattering cross section should decrease as $\epsilon_{00}(H)=\epsilon_{00}(0)-$ $C_{1}\left(H / H_{c 2}^{0}\right) \ln \left(H_{c 2}^{0} / H\right)$ along the vertical arrow in Fig. 1; (iii) when starting from the SC $+\mathrm{SDW}$ phase at $H=0$, one finds an increase of the elastic neutron scattering given by $I(H)=I(0)+C_{2}\left(H / H_{c 2}^{0}\right) \ln \left(H_{c 2}^{0} / H\right)$, where $C_{1}$ and $\mathrm{C}_{2}$ are some constants. All these functional forms are expected to be exact at small $H$; naively, one might have expected an analytic series in powers of $\vec{H}^{2}$, but the infinite diamagnetic susceptibility of a superconductor replaces this with a nonanalytic function of $|\vec{H}|$. We will also discuss the case of $\kappa$ large but finite.

Because the SC order is present on both sides of the transition, we can describe it in a static GL theory: the free energy $F_{\mathrm{GL}}$ is given by

$$
\begin{gathered}
\int d^{2} x\left[-|\psi|^{2}+\frac{|\psi|^{4}}{2}+\left|\left(\vec{\nabla}_{x}-i \vec{A}\right) \psi\right|^{2}\right. \\
\left.+\kappa^{2}\left|\vec{\nabla}_{x} \times \vec{A}\right|^{2}\right] .
\end{gathered}
$$

Here $\psi(x)$ is the complex SC order parameter, and we have performed standard rescalings to cast this theory in a dimensionless, universal form: all lengths $(x)$ are measured in units of the (bare) superconducting coherence length $\xi$, the vector potential is scaled as $\vec{\nabla}_{x} \times \vec{A}=\left(H / H_{c 2}^{0}\right) \hat{z}$, and energies are measured in units of $H_{c}^{2} \xi^{2} /(4 \pi)\left(H_{c}\right.$ is the field at which the free energy of the uniform superconductor equals that of the normal state). When $\kappa \rightarrow \infty$, as is the situation in the experiments on the cuprates [1], the screening of $H$ by the supercurrents is negligible, and we can develop our theory for the SC to SC + SDW transition taking $H$ to be uniform. We will not use any particular model for the dynamics of $\psi$, apart from assuming that its slow evolution occurs on time scales longer than those of the spin fluctuations of interest, and is presumably associated with the thermal and quantum motion of vortices [16].

The SDW order parameter is a $N=3$ component vector, $\phi_{\alpha}(x, \tau)$, where $\alpha=1, \ldots, N$ and $\tau$ is imaginary time. The quantum fluctuations of the $\phi_{\alpha}$ as a function of $\tau$ are known to play an important role even in the insulating antiferromagnet, and must surely be included in the vicinity of a quantum transition in a low dimensional magnet. For simplicity, we will assume that $\phi_{\alpha}$ is real, but our theory admits a simple generalization to incommensurate ordering transitions requiring a complex order parameter [11]. The dynamics of $\phi_{\alpha}$ is described by the action [17] (in the same units as $F_{\mathrm{GL}}$ ):

$$
\begin{aligned}
& S=\int d^{2} x \int_{0}^{1 / T} d \tau\{ \frac{1}{2}\left\{\left(\partial_{\tau} \phi_{\alpha}\right)^{2}+c^{2}\left(\nabla_{x} \phi_{\alpha}\right)^{2}\right. \\
&\left.+\left[r+v|\psi(x)|^{2}\right] \phi_{\alpha}^{2}\right\} \\
&\left.+\frac{u}{2}\left(\phi_{\alpha}^{2}\right)^{2}\right\},
\end{aligned}
$$

where the $x, \tau$ dependence of $\phi_{\alpha}$ is implicit, $\psi(x)$ is $\tau$ independent as discussed above, and $c, r, v, u$ are coupling constants, with $v^{2}<4 u$. We have neglected fermionic excitations because momentum conservation constraints suppress their coupling to $\phi_{\alpha}$ [11]. The action is the same as that near SDW ordering transitions in insulators, with the simplest symmetry-allowed terms in powers of $\phi_{\alpha}$ and spatial and temporal gradients. The tuning parameter $r$ (which, presumably, increases monotonically with increasing doping, $\delta$ ) drives the theory from the SC + SDW phase (with $\left\langle\phi_{\alpha}\right\rangle \neq 0,\langle\psi\rangle \neq 0$ ) to the SC phase (with $\left\langle\phi_{\alpha}\right\rangle=0,\langle\psi\rangle \neq 0$ ) with increasing $r$; at $H=0, T=0$, this transition is at $r=r_{c}$ (Fig. 1). The latter phase has a $S=1$ exciton (or a collective "spin resonance") associated with oscillations of $\phi_{\alpha}$ about $\phi_{\alpha}=0$, with an energy which vanishes as $r \searrow r_{c}$ [18]. Our main results for the $H$ dependence of the physics in the vicinity of the SC to SC + SDW phase boundary depend crucially on the coupling $v>0$ between the amplitudes of the SC and SDW orders; such a repulsive coupling was emphasized in [6,9].

A powerful tool to account for the quantum fluctuations of $\phi_{\alpha}$ is the large $N$ expansion [18]. At large $N$ and small $T$, the saddle-point equations describing the properties of $S+F_{\mathrm{GL}} / T$ in phases with $\left\langle\phi_{\alpha}\right\rangle=0$ are

$$
\begin{gathered}
\mathcal{V}(x)=r+v|\psi(x)|^{2}+2 N u T \sum_{\omega_{n}} G\left(x, x, \omega_{n}\right), \\
{\left[-1+|\psi(x)|^{2}-\left(\vec{\nabla}_{x}-i \vec{A}\right)^{2}\right] \psi(x)+} \\
(N v T / 2) \sum_{\omega_{n}} G\left(x, x, \omega_{n}\right) \psi(x)=0,
\end{gathered}
$$

where $\omega_{n}$ is a Matsubara frequency and $G\left(x, x^{\prime}, \omega_{n}\right) \delta_{\alpha \beta}=\int_{0}^{1 / T} d \tau e^{i \omega_{n} \tau}\left\langle\phi_{\alpha}(x, \tau) \phi_{\beta}\left(x^{\prime}, 0\right)\right\rangle \quad$ is the $\phi_{\alpha}$ Green's function which obeys

$$
\left[\omega_{n}^{2}-c^{2} \vec{\nabla}_{x}^{2}+\mathcal{V}(x)\right] G\left(x, x^{\prime}, \omega_{n}\right)=\delta^{2}\left(x-x^{\prime}\right) .
$$

The solution of (2), (3), and (4) (and their straightforward generalization to phases with $\left\langle\phi_{\alpha}\right\rangle \neq 0$ [18]) leads to the phase diagram in Fig. 1. We emphasize that the present model is accurate only at small $H$ in the vicinity of the $\mathrm{SC}$ to SC + SDW transition. The other phases in Fig. 1 involve loss of SC order, and for these a more complete treatment of the charge fluctuations is surely needed: various site- and bond-centered charge ordered states ("stripes" and "spin-Peierls") and Wigner crystal states are likely to play a significant role (see, e.g., [11,14]).

We now present an analytical description of the crucial physical properties of the solution of (2), (3), and (4) in the SC phase at small $H$ and close to $r_{c}$; a full numerical solution will be presented in future work. The SC order, $\psi$, supports an Abrikosov flux lattice of vortices at $N_{v} \rightarrow \infty$ positions $\left\{R_{v}\right\}\left[\psi\left(R_{v}\right)=0\right]$, with a core size of order unity, and a lattice spacing $L_{v} \sim \sqrt{H_{c 2}^{0} / H}$. The resulting $|\psi(x)|^{2}$ acts like a periodic potential for $\phi_{\alpha}$, and it is useful express $G$ in terms of the Bloch states, $g_{n k}(x)=$ $u_{n}(x) e^{i k x}$ of this periodic potential 


$$
G\left(x, x^{\prime}, \omega_{n}\right)=\frac{1}{N_{v}} \sum_{n k} \frac{g_{n k}^{*}(x) g_{n k}\left(x^{\prime}\right)}{\omega_{n}^{2}+\epsilon_{n k}^{2}},
$$

where $\left[-c^{2} \vec{\nabla}_{x}^{2}+\mathcal{V}(x)\right] g_{n k}(x)=\epsilon_{n k}^{2} g_{n k}(x), \quad n$ is a "band" index, $k$ extends over the first Brillouin zone of the reciprocal lattice of $\left\{R_{v}\right\}$, and the eigenvalues $\epsilon_{n k}^{2}>0$; SDW order appears when one of $\epsilon_{n k}$ first vanishes. An immediate experimental consequence of this structure is the presence of "Bragg reflections" at wave vectors separated by the reciprocal lattice vectors in the dynamic spin structure factor, as shown in Fig. 2.

Our interest will primarily be in the nature of the lowest energy band, $\epsilon_{0 k}$, which also controls the transition to the $\mathrm{SC}+\mathrm{SDW}$ state. As the cores of the vortices act as attractive potentials for $g_{n k}(x)$, a possibility for such a state is a superposition of Wannier orbitals localized at the cores of the vortices [6]: $g_{0 k}(x)=\sum_{\left\{R_{v}\right\}} e^{i k R_{v}} f\left(x-R_{v}\right)$ where $f(x)$ is exponentially localized on a scale $\ell \ll L_{v}$. However, the self-interactions of the $\phi_{\alpha}$, accounted for by the self-consistent potential proportional to $u$ in (2), have a large impact on these localized states, and we will establish that such a structure for $g_{0 k}$ must break down as the transition to the onset of SDW order is approached. The key argument was made by Bray and Moore [19] in a different physical context, and we review their reasoning. As we are assuming $\ell \ll L_{v}$, the localized states at the vortex cores can be treated independently of each other: $\epsilon_{0 k}$ is independent of $k$ and $|f(0)| \sim 1 / \ell$. Let $\mathcal{V}(x)=\mathcal{V}_{>}\left[\boldsymbol{V}(x)=\mathcal{V}_{<}\right]$for $x$ outside [inside] the vortex cores where $\psi(x) \approx 1[\psi(x) \approx 0]$. Then subtracting (2) evaluated at these $x$ 's from each other, and noting that the difference in the term proportional to $u$ arises primarily from the localized state in the vortex core, we obtain (at $T=0$ ) $v-N u|f(0)|^{2} / \epsilon_{00} \approx V_{>}-V_{<}>0$. This implies that the localization length must be at least as large

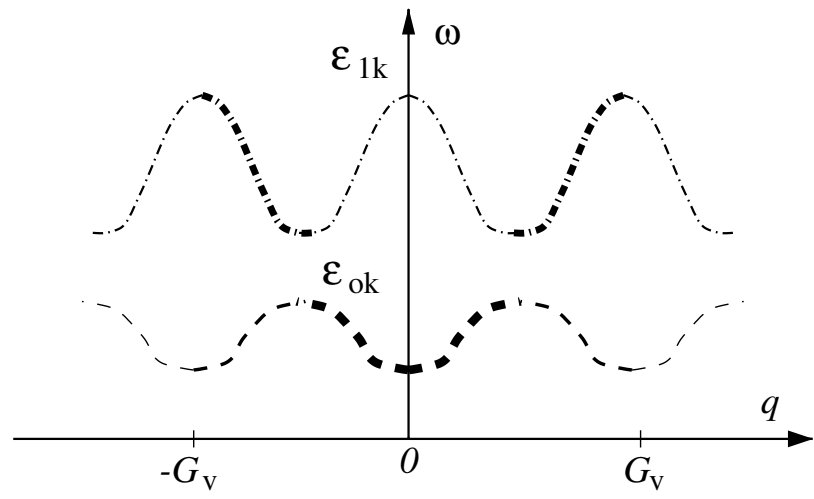

FIG. 2. Dynamic spin susceptibility in the vortex state $\chi^{\prime \prime}(q, \omega)=\operatorname{Im}\left[\int_{x, x^{\prime}} e^{i q\left(x-x^{\prime}\right)} G\left(x, x^{\prime}, \omega\right)\right]=\sum_{n, G m} \int_{k}\left|u_{n}\left(G_{m}\right)\right|^{2}$ $\epsilon_{n k}^{-1} \delta\left(k+G_{m}-q\right) \delta\left(\omega-\epsilon_{n k}\right), \quad k$ integration is over the Brillouin zone of the reciprocal lattice of $\left\{R_{v}\right\}, n$ is a band index, $G_{m}$ are reciprocal lattice vectors, and $u_{n}\left(G_{m}\right)$ are Fourier transforms of the periodic wave functions $u_{n}(x)$. Note that dispersing $S=1$ exciton modes $\delta\left(\omega-\epsilon_{n k}\right)$ are shifted by all $G_{m}$ with the weight $\left|u_{n}\left(\mathbf{G}_{m}\right)\right|^{2}$. as $\ell \sim 1 / \sqrt{\epsilon_{00}}$. As $\epsilon_{00} \searrow 0$ upon approaching the onset of SDW order, we must eventually have $\ell \sim L_{v}$, and the assumed structure for $g_{0 k}$ breaks down.

We are, therefore, compelled to turn our attention to extended, plane-wave-like wave functions for the $g_{n k}$ (we do not exclude nontrivial structure in these wave functions on the scale $L_{v}$ ). The self-interaction terms are now expected to be less important: we initially neglect the terms proportional to $G$ in (2) and (3), but will account for them later. We now demonstrate that, for small $H$, the eigenenergies, $\epsilon_{0 k}$, are controlled by universal structure in $\psi(x)$ in the region $1 \ll|x| \ll L_{v}$ well outside the vortex core at $R_{v}=0$ (and in the corresponding regions of the other vortices). Analysis of (3), following the standard description of a superconducting vortex, shows that in such regions

$$
|\psi(x)|=1-1 /\left(2 x^{2}\right) ;
$$

the second term above is the correction in the amplitude of the SC order induced by the superflow kinetic energy. We now use perturbation theory to evaluate the change in $\epsilon_{00}$ induced by (6); this requires the quantity

$$
\left\langle|\psi(x)|^{2}\right\rangle_{x}=1-\left[H /\left(2 H_{c 2}^{0}\right)\right] \ln \left(\vartheta_{2} H_{c 2}^{0} / H\right),
$$

where the average is over spatial positions $x$, the logarithm arises from the integral of $1 / x^{2}$ over the two-dimensional unit cell of the vortex lattice (it is cut off at short scales by the superconducting coherence length $\sim 1$, and at long scales by $L_{v}$ ), and the constant $\vartheta_{2} \approx 3$ (for triangular and square vortex lattices) was obtained by numerical solution of (3) using the method of [20]. From (2), we obtain the leading perturbative correction

$$
\epsilon_{00}^{2}(H)=\epsilon_{00}^{2}(0)-\left[v H /\left(2 H_{c 2}^{0}\right)\right] \ln \left(\vartheta_{2} H_{c 2}^{0} / H\right) ;
$$

this result is also a variational upper bound on $\epsilon_{00}(H)$, associated with the wave function $g_{00}=1$. For small $r-r_{c}$ we have $\epsilon_{00}^{2}(0)=\left(r-r_{c}\right)$ (this corresponds to the exponent $\nu=1 / 2$ in mean field theory), and so we can rewrite (8) as $\epsilon_{00}(H)=\left[r(H)-r_{c}\right]^{1 / 2}$ where

$$
r(H) \equiv r-\left[v H /\left(2 H_{c 2}^{0}\right)\right] \ln \left(\vartheta_{2} H_{c 2}^{0} / H\right) .
$$

The vanishing of $\epsilon_{00}(H)$ determines the position of the phase boundary AM between the SC and SC + SDW phases at small $H$ :

$$
H / H_{c 2}^{0}=2\left(r-r_{c}\right) /\left\{v \ln \left[1 /\left(r-r_{c}\right)\right]\right\} ;
$$

the variational argument shows that this is an upper bound for $H$, and so the phase boundary can become flatter only at higher orders. A fully self-consistent calculation which includes the terms proportional to $G$ in (2) and (3) is more involved, but tractable: we find a modified functional dependence of $\epsilon_{00}(H)$ on $r(H), \epsilon_{00}(H)=$ $2 \pi c^{2}\left[r(H)-r_{c}\right] /\left[N u\left(1-v^{2} / 4 u\right)\right]$ (corresponding to the exponent $\nu=1$ in the large $N$ limit [18]), but the expressions (9) and (10) continue to hold.

We comment further on experimental implications of our results. For very small $H$, if $g_{0 k}$ is localized on a scale $\ell \ll L_{v}$, the lowest energy magnetic mode is a 


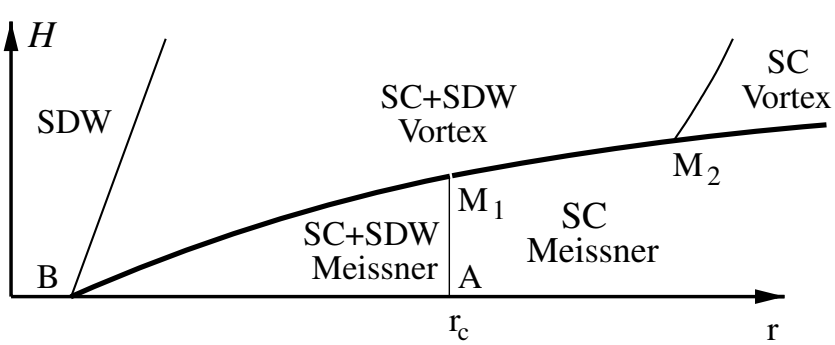

FIG. 3. Phase diagram of $S+F_{\mathrm{GL}} / T$ for finite $\kappa$ and small fields. The SC and SC + SDW phases in Fig. 1 are identical to the corresponding "vortex" phases here. The position of $H_{c 1}$ is given by $H_{c 1} / H_{c 2}^{0}=\sqrt{\pi / 2}\left(\ln \kappa / \kappa^{2}\right) \psi_{H=0}^{2}$, where for the $\mathrm{BM}_{1}$ line $\psi_{H=0}^{2}=1-v\left(r_{c}-r\right) /\left[4 u\left(1-v^{2} / 4 u\right)\right]$, and for the $\mathrm{M}_{1} \mathrm{M}_{2}$ line $\psi_{H=0}^{2}=1+N v \epsilon_{00}(0) / 8 \pi$ with $\epsilon_{00}$ discussed after Eqs. (8) and (10).

relatively high energy $S=1$ excitonic mode near the vortex cores, as noted earlier. The spectral weight of this mode will be much smaller than the slightly higher energy bulk contribution from outside the vortex cores, and will consist of a feature of width $\delta k=\ell^{-1}$ (resolution of the Bragg reflections may not be feasible). For slightly larger $H$, the distinction between the localized and delocalized contributions will disappear, and we expect that a full dynamic spin structure with Bragg reflections may be resolved in neutron scattering experiments, with the lowest energy mode obeying (8). It is also interesting to note that, even for small $H$, repulsion between the SC and SDW order parameters described by the $v$ term in (1) will lead to an interesting structure in the spatial form of $|\psi(x)|$ on the scale $\ell$ which may be significantly larger than the bare SC coherence length (unity in our units). This surprising effect, where the superconducting vortex core structure depends on the magnetic field for $H \ll H_{c 2}^{0}$, is another significant prediction of our theory, and is testable, e.g., in tunneling experiments.

An interesting recently observed effect [2,3] may readily be accounted for by $S+F_{\mathrm{GL}} / T$ : the intensity of the elastic neutron scattering increases when $H$ is applied to the SC + SDW state (line BA on Fig. 1). In the large $N$ limit we find that the expectation value of the staggered moment increases with $H$ as $\left\langle\left|\phi_{\alpha}\right|\right\rangle^{2}=\left[r_{c}-r(H)\right] /$ $\left[2 u\left(1-v^{2} / 4 u\right)\right]$, with $r(H)$ given in (9). Quantitative agreement with this relation has been recently observed [4].

We now discuss the consequences of supercurrent screening of the magnetic field at finite $\kappa$. For finite $\kappa$ and small $H$ we will have a Meissner phase where no vortices penetrate the sample. As shown in Fig. 3, we may have Meissner phases that are purely superconducting or have coexisting magnetism. It is interesting to note that a finite density of vortices penetrates a sample at $H_{c 1}$, so there is a finite jump in the SDW order across the lines $\mathrm{BM}_{1}$ and $\mathrm{M}_{1} \mathrm{M}_{2}$.

We conclude by noting some broader implications of our results. (i) In the discussion above we assumed that $v>0$, which is consistent with the experimental situation in $\mathrm{La}_{2-\delta} \mathrm{Sr}_{\delta} \mathrm{CuO}_{4}$. The case $v<0$, where the transition into the SC state leads to an enhancement in the SDW fluctuations, may also be described following the formalism above. (ii) Closely related phase diagrams should apply to other ordering transitions of superconductors in a field, including charge density wave and "staggered flux" orders.

This research was supported by NSF Grant No. DMR 0098226 (Yale), and by the Harvard Society of Fellows. We acknowledge useful discussions with G. Aeppli, R. Birgeneau, B. Khaykovich, M. Kastner, S. Kivelson, Y. Lee, and S.-C. Zhang.

[1] B. Lake et al., Science 291, 1759 (2001).

[2] S. Katano et al., Phys. Rev. B 62, R14 677 (2000).

[3] B. Lake et al., cond-mat/0104026.

[4] B. Khaykovich et al. (unpublished); B. Lake et al. (unpublished).

[5] S. Sachdev, Phys. Rev. B 45, 389 (1992); N. Nagaosa and P. A. Lee, ibid. 45, 966 (1992); M. Franz and Z. Tesanovic, ibid. 63, 064516 (2001).

[6] D. P. Arovas et al., Phys. Rev. Lett. 79, 2871 (1997).

[7] P. Hedegard, cond-mat/0102070.

[8] M. Inui et al., Phys. Rev. B 37, 2320 (1988).

[9] S.-C. Zhang, Science 275, 1089 (1997).

[10] L. Balents, M.P. A. Fisher, and C. Nayak, Int. J. Mod. Phys. B 12, 1033 (1998).

[11] M. Vojta and S. Sachdev, Phys. Rev. Lett. 83, 3916 (1999); M. Vojta, C. Buragohain, and S. Sachdev, Phys. Rev. B 61, 15152 (2000).

[12] J. H. Han, Q.-H. Wang, and D.-H. Lee, cond-mat/0006046.

[13] I. Martin et al., Int. J. Mod. Phys. 14, 3567 (2000).

[14] M. Granath et al., cond-mat/0010350.

[15] Y.S. Lee et al., Phys. Rev. B 60, 3643 (1999).

[16] There is also a fast mode of $\psi$ oscillations at frequencies of order the plasma frequency - it is assumed that this mode merely renormalizes the parameters of the critical modes of interest here.

[17] The spin Zeeman coupling, under which $\partial_{\tau} \phi_{\alpha} \rightarrow \partial_{\tau} \phi_{\alpha}-$ $i \epsilon_{\alpha z \beta} H \phi_{\beta}$, leads to weaker effects of order $H^{2}$ which we neglect.

[18] A. V. Chubukov, S. Sachdev, and J. Ye, Phys. Rev. B 49, 11919 (1994).

[19] A. J. Bray and M. A. Moore, J. Phys. C 15, L765 (1982); J. W. Hartman and P. B. Weichman, Phys. Rev. Lett. 74, 4584 (1995).

[20] E. Brandt, Phys. Rev. Lett. 78, 2208 (1997).

[21] We also shift the coefficient of $|\psi|^{2}$ in $F_{\mathrm{GL}}$ by $(N v / 2) \times$ $\int d^{2} k d \omega /\left[8 \pi^{3}\left(\omega^{2}+c^{2} k^{2}\right)\right]$ to renormalize away the zero-point fluctuations in $\phi_{\alpha}$ at $r=r_{c}, T=0, H=0$. 